\title{
Multi-Time Scale Perspective in Analyzing Cardiovascular Data
}

\author{
H. K. LACKNER ${ }^{1,2 *}$, J. J. BATZEL ${ }^{1}$, A. RÖSSLER ${ }^{1}$, H. HINGHOFER-SZALKAY ${ }^{1}$, \\ I. PAPOUSEK ${ }^{2 *}$ \\ *These authors contributed equally to the paper.
}

${ }^{1}$ Institute of Physiology, Medical University of Graz, Graz, Austria, ${ }^{2}$ Department of Psychology, Biological Psychology Unit, Karl-Franzens University, Graz, Austria

Received June 29, 2013

Accepted January 10, 2014

On-line April 3, 2014

\section{Summary}

Cardiovascular dynamic and variability data are commonly used in experimental protocols involving cognitive challenge. Usually, the analysis is based on a sometimes more and sometimes less well motivated single specific time resolution ranging from a few seconds to several minutes. The present paper aimed at investigating in detail the impact of different time resolutions of the cardiovascular data on the interpretation of effects. We compared three template tasks involving varying types of challenge, in order to provide a case study of specific effects and combinations of effects over different time frames and using different time resolutions. Averaged values of hemodynamic variables across an entire protocol confirmed typical findings regarding the effects of mental challenge and social observation. However, the hemodynamic response also incorporates transient variations in variables reflecting important features of the control system response. The fine-grained analysis of the transient behavior of hemodynamic variables demonstrates that information that is important for interpreting effects may be lost when only average values over the entire protocol are used as a representative of the system response. The study provides useful indications of how cardiovascular measures may be fruitfully used in experiments involving cognitive demands, allowing inferences on the physiological processes underlying the responses.

\section{Key words}

Cardiovascular reactivity - Transient response - Cognitive challenge $\bullet$ Social observation $\bullet$ Stress

\section{Corresponding author}

H. K. Lackner, Medical University of Graz, Institute of Physiology,
Harrachgasse 21/V, A-8010 Graz, Austria. E-mail: helmut.lackner @medunigraz.at

\section{Introduction}

The interaction of the cardiovascular system with other neural and psychoneural pathways provides a unique window for assessing individual cardiovascular functional effects as well as insight into the pathways of interaction between systems (Berntson et al. 1996, Lovallo 1997, Lackner et al. 2013, Papousek et al. 2013). However, these interactions do not only present opportunities but also create subtle issues and complications in regards to the interpretation of effects (Parati et al. 1995, Kamarck and Lovallo 2003, Lackner et al. 2010a).

While most researchers are aware of the importance of specific time scales in the analysis and interpretation of data, they rarely make use of the possibilities offered by the use of multiple time scales in the analysis of a given data set. The availability of stateof-the-art equipment in most labs, allowing highly synchronous recordings of several physiological variables including electrocardiographic, hemodynamic and other variables, makes the multi-time scale perspective especially valuable. Therefore, in this study we will develop a coordinated examination of three template tasks of increasing level of complexity utilizing several time scales of data analysis. The goal is to demonstrate how incorporating different levels of time scale allows for a more specific analysis of the factors that can shape the characterization of responses to tasks. 
In psychophysiological experiments, cardiovascular dynamic and variability data have been used to address a variety of research questions in the context of higher cognitive processes.

There is an extensive literature addressing the influence of motivational factors, such as incentive or the perceived task difficulty on cardiovascular responses to cognitive challenge (e.g. Callister et al. 1992, Eubanks et al. 2002, Iani et al. 2004, Richter and Gendolla 2007, Richter et al. 2008, Seery et al. 2009, Silvestrini and Gendolla 2009). Less attention has been paid to properties that are inherent in the task and which may exert notable influences on cardiovascular responses even though other factors are actually the focus of interest. The physical characteristics of the stimulus represent one pathway through which additional effects can be introduced. Moreover, as most experimental tasks involve not only cognitive activity, but also physical action and emotional stress, drawing clear conclusions requires a careful assessment of confounding factors and their cardiovascular effects.

The issue of stimulus properties has received much attention beginning with the seminal work of Graham and Clifton (1966). Specific stimulus characteristics have been shown to evoke specific cardiac responses such as orienting, startle, and defensive responses (Graham 1979, Turpin et al. 1999, Ramírez et al. 2005). But also the effects of the presentation of a stimulus as such and even the pace of the stimuli in the testing protocol should be considered when interpreting cardiovascular responses (Lackner et al. 2010a).

To assess the task performance, participants typically are required to respond either with a verbal response or nonverbally using some device such as a button or computer mouse. In addition to the presentation of a new stimulus and timing prompts, the response modality may also affect the cardiovascular variables during the performance of cognitive tasks, which can further complicate the interpretation of results (Lang et al. 1993, Codispoti et al. 2001, Lackner et al. 2010a).

Finally, psychophysiological experiments vary in the obviousness of observation and evaluation, introduced, for instance, by the presence of surveillance cameras or experimenters in the room with the participants, or by the task conditions. For example, overt responses may be required that are apparently monitored online by the experimenter. This places social-evaluative stress on the participants, which typically enhances physiological reactivity (Cacioppo et al. 1990, Wright et al. 1995, Al'Absi et al. 1997, Smith et al. 1997, Kelsey et al. 2000, Gendolla and Richter 2006, Gramer and Saria 2007).

For the most part, the experimental tasks typically employed in experiments involving cardiovascular responses to cognitive challenge can be described as active coping tasks (Obrist 1981). Such tasks are considered to evoke primarily cognitive activity, involving memory and close attention. Cardiovascular and hemodynamic responses to these tasks include an increase in arterial blood pressure, heart rate, and cardiac output as well as a decrease (or at least no increase) in systemic vascular resistance. More recent studies typically involved not only the recording of hemodynamic variables but also the calculation of hemodynamic variability. Beat to beat measurements of heart rate and blood pressure are used along with thoracic impedance to derive a continuous estimate of stroke volume, cardiac output and total peripheral resistance. In addition, the synchronisation of heart rate, blood pressure and respiration can provide a more detailed picture of the effect of mental challenge and the influence of respiration on the different branches of the autonomic nervous system (Lackner et al. 2011). The use of such sophisticated methods of measurement and analysis of cardiovascular data requires that great attention be given to the experimental set-up. This is important not only for drawing conclusions about the factors that are the focus of a particular research question but also for detecting subtle differences that may not be expected.

\section{Structure of the present study}

The aim of the present study was to investigate the impact of different time resolutions of the cardiovascular data on the interpretation of effects. In experimental studies using cardiovascular responses to cognitive challenges, the psychophysiological measures such as heart rate or blood pressure have typically been averaged over periods of at least three or five minutes as well as examined reactivity as the change from e.g. the last minute of baseline to the first minute of task. However, considering the time course of cardiovascular responses induced by mental challenge (e.g. Lackner et al. 2010b), essential effects may be more pronounced or may only become apparent by using higher time resolutions. Therefore, we studied (I.) the overall reactivity, that is, response patterns across overall time frames (300 s epochs) to confirm the expected effect of 
the tasks; (II.) transient responses step by step, that is, the time courses to the different challenges during the whole task period using time frames of $1 \mathrm{~min}$ referred to as transient overall response; (III.) transient response analyzing initial responses to the different challenges using time frames of $30 \mathrm{~s}$; and (IV.) $7.5 \mathrm{~s} \quad(7.5 \mathrm{~s}$ represents the time period in each trial) referred to as transient initial response. In addition (V.) transient responses, studying immediate responses to the stimulus presentation by $1.5 \mathrm{~s}$ frames analysis during the trials of $7.5 \mathrm{~s}$ each referred to as transient immediate response were calculated (see Fig. 1).

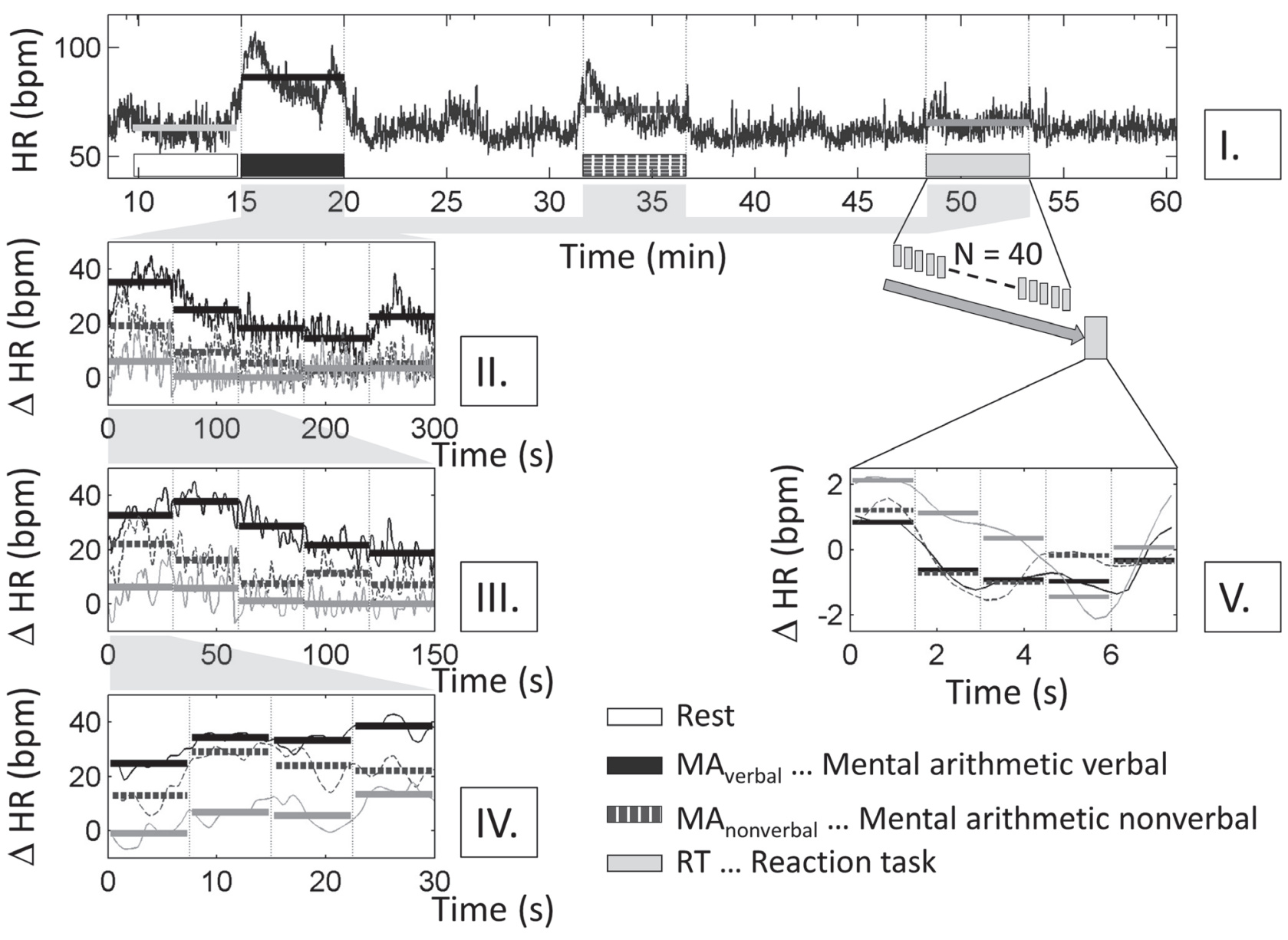

Fig. 1. Step by step analysis of heart rate for a single participant. Step [I.] shows the time course of the heart rate and the overall reactivity (average of $5 \mathrm{~min}$ epochs each; depicted by horizontal lines). Step [II.] shows the relative values compared to rest and the average of 1 min epochs (depicted by horizontal lines for the different tasks) for statistical use as well as the transient responses in the background. Step [III.] and step [IV.] demonstrate the calculation for a time resolution of $30 \mathrm{~s}$ and $7.5 \mathrm{~s}$. Step [V.] shows using the reaction task as an example the split of the heart rate values in 40 epochs (indicated by the screen change) of $7.5 \mathrm{~s}$ each relative to the mean of the $1.5 \mathrm{~s}$ frame directly preceding the screen change. The resulting time course were averaged for epochs of $1.5 \mathrm{~s}$ (see lines in the lower right panel) for further statistical use.

Using these five different time resolutions we investigated in detail the impact of variations in the experimental set-up on the collected cardiovascular data and their interpretation. The cardiovascular responses were compared for (a) simple reaction tasks, (b) mental arithmetic task requiring nonverbal responses and (c) mental arithmetic tasks requiring verbal responses.

The simple reaction task represented a psychomotor stressor posing only minimal cognitive demands whereas the nonverbal variety of the mental arithmetic task represented a cognitive stressor, but had the same motor demands as the simple reaction task. Finally, as compared to the nonverbal task, where the responses were given in private by selecting the correct answer on the computer monitor, the verbal mental arithmetic task implied an additional social-evaluative element. We analyzed differences between types of task (simple reaction task, mental arithmetic task) and types of responses (motor, verbal), and will demonstrate how the various effects that make up the cardiovascular system 
response to a cognitive task may be disentangled and interpreted by analyzing the effects of a particular task relative to an appropriate reference condition and using different time resolutions of data analysis.

In summary, using changes in time resolutions in a step by step procedure, the study will provide indications of how cardiovascular measures may be fruitfully used in psychophysiological experiments, allowing inferences on the physiological processes underlying the responses.

\section{Methods}

\section{Participants}

Sixty-two participants completed the experiment. Due to the strict artefact handling, four participants were excluded from the analysis. The final sample was comprised of 58 participants (29 men, 29 women) aged 19 to 53 years $(M=24.3, S D=6.1)$. Participants were requested to refrain from alcohol for twelve hours and from coffee and other stimulating beverages for four hours prior to their lab appointment, and to come to the session well rested. No participant reported using drugs or medication that may alter cardiovascular activity, and none had cardiovascular problems, chronic metabolic disease, or psychological disorders according to self-report. The study was performed in accordance with the 1964 Declaration of Helsinki and was approved by the local ethics committee. Written informed consent was obtained from all participants.

\section{Task protocols}

Each of the three tasks was comprised of 40 trails, presented at a space of $7.5 \mathrm{~s}$ per trail.

\section{Mental arithmetic task verbal (MA $\left.A_{\text {verbal }}\right)$}

The items of this task consisted of two pairs of one-digit numbers, which were to be subtracted or added (e.g. $5-2 ; 2+6$ ). The two sums had to be memorized and processed according to the following rules: 1) If the first sum was higher than the second sum, the second sum had to be subtracted from the first sum. 2) If the first sum was lower than the second sum, the second sum had to be added to the first sum. Participants were asked to work as fast and exactly as possible, to answer aloud within the given time frame $(7.5 \mathrm{~s}$ in each trial), and were informed that the experimenter will record their answer (thereby inducing an additional social-stress component). The tasks were presented consecutively on a computer screen. A counter indicated the remaining time for delivering the answer, and during the last $1.5 \mathrm{~s}$ the grey rectangles forming the background of the numbers changed to red.

\section{Mental arithmetic task nonverbal (MA $\left.A_{\text {nonverbal }}\right)$}

In difference to the screen display used in the $\mathrm{MA}_{\text {verbal }}$ task, three buttons with possible solutions of the arithmetic problems were arranged on the right hand side of the screen. The participants were asked to work as fast and exactly as possible, and to click on the correct button using the computer mouse within the given time frame (7.5 s each) instead of answering aloud.

\section{Reaction task (RT)}

In this task only grey rectangles at the place of MA tasks were initially displayed on the screen, which changed to red after $6 \mathrm{~s}$. One out of the three buttons of the $\mathrm{MA}_{\text {nonverbal }}$ was visible on the right hand side of the screen during the last $1.5 \mathrm{~s}$ of a trial, which had to be clicked as fast as possible during the remaining time.

\section{Recording of physiological variables}

Continuous hemodynamic monitoring of blood pressure $\left(\mathrm{BP}\right.$; sampling rate $=100 \mathrm{~Hz}, \mathrm{BP}_{\text {range }}=$ $50-250 \mathrm{~mm} \mathrm{Hg}, \pm 5 \mathrm{~mm} \mathrm{Hg}$ ), heart rate (HR; 3-lead electrocardiography (ECG), sampling rate $=1 \mathrm{kHz}$, $\mathrm{f}_{\text {cut-off }}=0.08-150 \mathrm{~Hz}$ ) and thoracic impedance (sampling rate $=100 \mathrm{~Hz}, \mathrm{Z}_{0 \text {,range }}=10-75 \Omega, \mathrm{dZ} / \mathrm{dt}= \pm 10 \Omega / \mathrm{s}$ ) were carried out with the Task Force Monitor ${ }^{\circledR}$ (TFM ${ }^{\circledR}$; CNSystems, Graz, Austria). Continuous blood pressure was derived from the finger using a refined version of the vascular unloading technique and corrected to absolute values with oscillometric blood pressure measurement on the contralateral upper arm by the TFM® (Fortin et al. 2006b). Electrodes were placed at the neck and thoracic regions, the latter specifically at the midclavicular line at the xiphoid process level (Fortin et al. 2006a).

\section{Procedure}

After arriving at the laboratory, the participants were seated in an acoustically shielded examination chamber. They were familiarized with the test protocol, equipment and personnel, and electrodes were attached. The non-dominant arm was placed on a pad at the level of the heart, to avoid effects of hydrostatic pressures on blood pressure values. Afterwards, they received instructions for the tasks. Before the first task was started, the physiological variables were recorded from $15 \mathrm{~min}$ 
prior to the task onset until $15 \mathrm{~min}$ and a $300 \mathrm{~s}$ recording (from $315 \mathrm{~s}$ till $15 \mathrm{~s}$ prior the task onset) was taken as rest period. Following each task, a $300 \mathrm{~s}$ rest period was observed, after which the participants were asked as an indicator of their motivation how much effort they had made to accomplish the task (17-point rating scale, from 1 "not at all" to 17 "extremely"). They were also asked to indicate how difficult they had experienced the task to be (17-point rating scale, from 1 "not at all" to 17 "extremely"). The scales had been used in previous investigations (e.g. Papousek et al. 2011). Before the next task was started, the continuous blood pressure recording was re-calibrated $(120 \mathrm{~s})$ and another $180 \mathrm{~s}$ rest period was observed. Each task protocol lasted for $300 \mathrm{~s}$. The order of tasks (RT, MA $\left.A_{\text {nonverbal, }} \mathrm{MA}_{\text {verbal }}\right)$ was counterbalanced. The recordings were controlled by a fully automated software development and each interaction of the participants with the PC was saved synchronously to the cardiovascular data. During the recordings, the experimenter was outside the examination chamber. Throughout the whole procedure the participants were observed through a one-way window and an intercom, and the signals were monitored online to ensure the signal quality. The participants remained seated during the whole procedure.

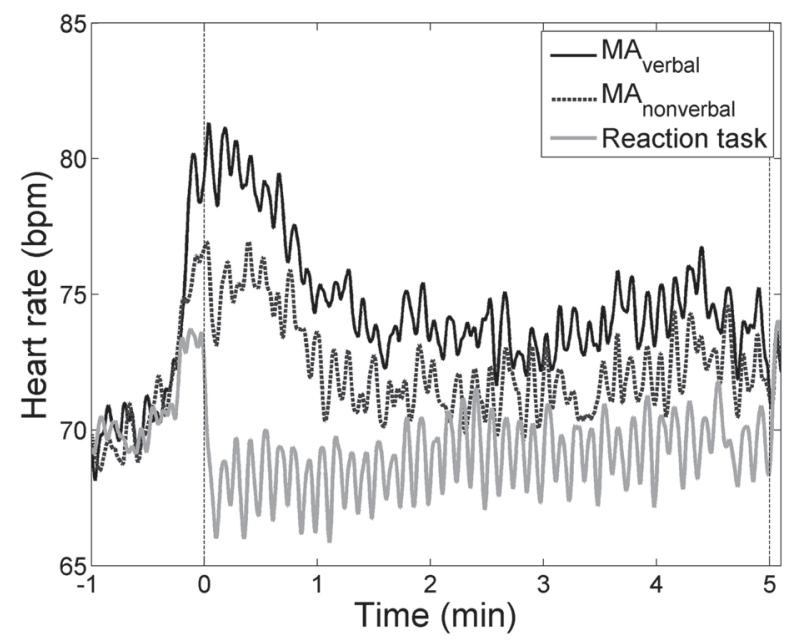

\section{Data reduction and analysis}

For heart rate, blood pressure and the variables related to impedance cardiography beat-to-beat values computed by the TFM ${ }^{\circledR}$ were used. Thoracic impedance $\mathrm{Z}_{0}(\mathrm{t})$ and impedance variation $\mathrm{dZ}(\mathrm{t}) / \mathrm{dt}$ were used to calculate beat-to-beat stroke volume based on an improved Kubicek approach and cardiac output (Gratze et al. 1998). Total peripheral resistance (TPR) was calculated as $80 \mathrm{x}$ (mean arterial blood pressure - central venous pressure)/cardiac output in which the central venous pressure was fixed at $7 \mathrm{~mm} \mathrm{Hg}$ (Gratze et al. 1998). The respiratory signal was derived from the raw data of thoracic impedance (Ernst et al. 1999, Houtveen et al. 2006).

Single artefacts were replaced by interpolation and their appearance recorded. Means of beat to beat values of HR, SV, CO, TPR, SBP, DBP and MAP and the mean and standard deviation of respiration (RF, $\mathrm{SD}_{\mathrm{RF}}$ ) were computed across the $300 \mathrm{~s}$ epochs. To obtain heart rate and blood pressure time series with equidistant time steps, the beat to beat values were resampled with $4 \mathrm{~Hz}$ using piecewise cubic spline interpolation after artefact correction. The resulting time course of HR and MAP during the different stressors applied can be seen in Figure 2.

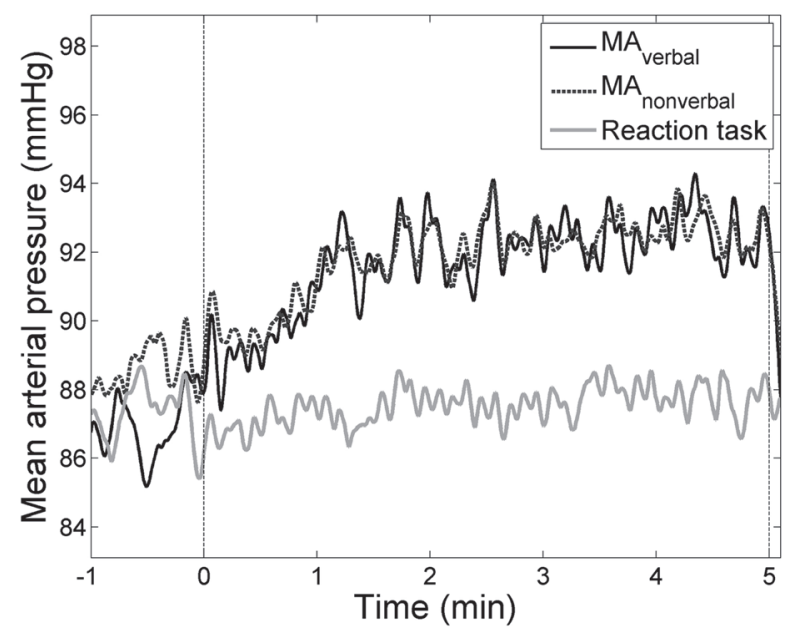

Fig. 2. Transient responses of heart rate and mean arterial pressure during the processing of the task protocols, averaged across participants. The dotted line at point 0 represent the begin of the task protocols (presented on the screen).

To investigate transient responses, stroke volume, cardiac output and total peripheral resistance were also resampled to $4 \mathrm{~Hz}$. The time domain variables of heart rate variability (HRV) and blood pressure variability (BPV) were computed as the standard deviation (SDNN, standard deviation of normal-tonormal beat; $\mathrm{SD}_{\mathrm{SBP}}$; and $\mathrm{SD}_{\mathrm{DBP}}$ ) across the $300 \mathrm{~s}$ epochs.
For frequency domain variables of R-R intervals (RRI), SBP, and DBP, Fourier Transform with a Hanning window after resampling and removing the trend of 2 nd order was used. Low frequency (LF) was defined as 0.04-0.15 Hz, high frequency (HF; used for RRI only) was defined as $0.15-0.40 \mathrm{~Hz}$, according to published recommendations (Task Force 1996). Because of skewed 
distributions of the frequency domain variables, a natural logarithmic transformation $(\ln )$ was applied.

\section{Transient responses: overall responses to the stressors}

To assess the time courses of the different challenges, average values across 1 min frames from the task onset until the end of the task were calculated ( stress $_{\min 1}$, stress $_{\min 2}$, stress $_{\min 3}$, stress $_{\min 4}$, stress $_{\min 5} ; 1 \mathrm{~min}$ each; see Fig. 1 [II.]). Scores for HR, SV, CO, TPR, SBP, MAP, and DBP were computed relative to the mean of the rest period.

\section{Transient responses: initial responses to the stressors}

To assess the time courses of the initial responses to the different challenges, average values across $30 \mathrm{~s}$ frames from the task onset until $2.5 \mathrm{~min}$ after the start of the task were calculated ( $\operatorname{stress}_{30 \mathrm{~s}, 1}$, stress $_{30 \mathrm{~s}, 2}$, stress $_{30 \mathrm{~s}, 3}$, stress $_{30 \mathrm{~s}, 4}$, stress $_{30 \mathrm{~s}, 5} ; 30 \mathrm{~s}$ each; see Fig. 1 [III.]). In addition, for the first $30 \mathrm{~s}$ after task onset $7.5 \mathrm{~s}$ frames were calculated, that is, for the first four trials (stress $\mathrm{srial}_{\text {tr }}$, stress $_{\text {trial2 }}$, stress $_{\text {trial3 }}$, stress $_{\text {trial4 }} ; 7.5 \mathrm{~s}$ each; see Fig. 1 [IV.]).

Furthermore, average values across $30 \mathrm{~s}$ frames from $2.5 \mathrm{~min}$ after the start of the task until the end of the task were calculated ( stress $_{30 \mathrm{~s}, 6}, \quad$ stress $_{30 \mathrm{~s}, 7}, \quad$ stress $_{30 \mathrm{~s}, 8}$, stress $_{30 \mathrm{~s}, 9}$, stress $_{30 \mathrm{~s}, 10} ; 30 \mathrm{~s}$ each) to examine the prolonged responses during the different challenges.

Scores for HR, SV, CO, TPR, SBP, MAP, and DBP were computed relative to the mean of the rest period.

Transient responses: immediate responses to stimulus presentation

Each task was comprised of 40 trials, presented at a pace of $7.5 \mathrm{~s}$ per trial. To examine the effect elicited by the presentation of a new item (screen change; SC), average values across $1.5 \mathrm{~s}$ frames from the screen change (new item) until the $7.5 \mathrm{~s}$ following the screen change were computed, relative to the mean of the $1.5 \mathrm{~s}$ frame directly preceding the stimulus (post- $\mathrm{SC}_{1}$, post- $\mathrm{SC}_{2}$, post-SC $\mathrm{S}_{3}$, post-SC $\mathrm{S}_{4}$, post-SC $\mathrm{S}_{5}$; for $\mathrm{HR}, \mathrm{SV}, \mathrm{CO}$, TPR, SBP, MAP, and DBP; see Fig. 1 [V.]).

\section{Statistical analysis}

To evaluate the overall effects of the stressors on the cardiovascular variables, that is, the effect of the stressors compared to the resting condition per se and the differences between the stressors applied, repeated measures analyses of variance (ANOVAs) were conducted with Protocol (300 s rest period, $\mathrm{MA}_{\mathrm{verbal}}$, $\mathrm{MA}_{\text {nonverbal }}$, RT, within-subjects factor) as the independent variable. Greenhouse-Geisser corrections were used to adjust for non-sphericity of the variance-covariance matrices.

A two-tailed significance level of $\alpha=0.05$ was used for the analyses. Analyses were conducted using HR together with the hemodynamic variables (SV, CO, TPR), the blood pressure variables (SBP, MAP and DBP), the respiration frequency $\left(\mathrm{RF}, \mathrm{SD}_{\mathrm{RF}}\right)$, and heart rate variability ( $\mathrm{SDNN}, \mathrm{LF}_{\mathrm{RRI}}, \mathrm{HF}_{\mathrm{RRI}}$ and $\mathrm{LF} / \mathrm{HF}_{\mathrm{RRI}}$ ) as the dependent variables, respectively. Bonferroni correction for multiple comparisons yielded critical $\mathrm{p}$-values of $\mathrm{p}<0.013, \mathrm{p}<0.016, \mathrm{p}<0.025$, and $\mathrm{p}<0.013$, to indicate statistical significance in the analysis mentioned above, respectively.

Transient responses of the hemodynamic variables were analyzed using repeated measures ANOVAs with Protocol ( $\left.\mathrm{MA}_{\text {verbal }}, \mathrm{MA}_{\text {nonverbal, }} \mathrm{RT}\right)$ and Time as within-subjects factors. This was done for the overall responses, the initial responses to the stressors, and the prolonged responses while stressors applied to see if the effect remained in the second half of the stress application, as well as the immediate responses to stimulus presentation.

A two-tailed significance level of $\alpha=0.05$ was used for the analyses. Analyses for transient responses were conducted using HR together with the hemodynamic variables (SV, CO, TPR) and the blood pressure variables (SBP, MAP and DBP) as the dependent variables, respectively. Bonferroni correction for multiple comparisons yielded critical $p$-values of $p<0.013$ and $\mathrm{p}<0.016$ to indicate statistical significance, respectively.

Estimates of effect size are reported using partial eta-squared $\left(\eta_{p}{ }^{2}\right)$, which gives the proportion of variance a factor or interaction explains of the overall (effect + error) variance ${ }^{1}$.

\section{Results}

\section{Overall reactivity}

Heart rate and hemodynamic variables

The ANOVAs revealed a significant effect of Protocol for $\operatorname{HR}\left(F(2.2,124.0)=29.1, \mathrm{p}<0.001, \eta_{p}{ }^{2}=0.34\right)$, $\mathrm{CO}\left(F(2.1,118.3)=23.2, \mathrm{p}<0.001, \eta_{p}{ }^{2}=0.29\right)$, and TPR

\footnotetext{
1 Additionally entering sex and age in the analyses did not change the statistical results (i.e. significant results remained significant and nonsignificant results remained non-significant).
} 
$\left(F(2.4,134.6)=4.8, \mathrm{p}<0.01, \eta_{p}{ }^{2}=0.08\right)$ but not for $\mathrm{SV}$ $(F(2.1,118.1)=1.5$, ns. $)$. Means and results of Bonferroni corrected post-hoc tests are shown in Figure 3. Viewed across the entire protocol, the mental arithmetic tasks produced an activating effect which was greater for $\mathrm{MA}_{\text {verbal }}$ than for $\mathrm{MA}_{\text {nonverbal. Relative to the rest }}$ condition, the heart rate increased on average by $4.6 \mathrm{bpm}$ during $\mathrm{MA}_{\text {verbal, }}$, by $2.2 \mathrm{bpm}$ during $\mathrm{MA}_{\text {nonverbal }}$ and decreased by 1.4 bpm during the RT task.

\section{Blood pressure}

The ANOVAs revealed a significant effect of Protocol for SBP, MAP and DBP
(SBP, $\quad F(2.5,144.8)=7.0, \quad \mathrm{p}<0.001, \quad \eta_{p}{ }^{2}=0.11 ; \quad$ MAP, $F(2.6,146.8)=14.2, \quad \mathrm{p}<0.001, \quad \eta_{p}{ }^{2}=0.20 ; \quad$ DBP, $\left.F(2.6,146.2)=15.7, \mathrm{p}<0.001, \eta_{p}{ }^{2}=0.22\right)$. Systolic, diastolic and mean arterial blood pressure increased during the mental arithmetic tasks as compared to the rest condition, but did not differ between $\mathrm{MA}_{\text {verbal }}$ and $\mathrm{MA}_{\text {nonverbal. }}$ See Figure 3 for means and post-hoc tests. As compared to the rest condition, blood pressure increased on average by $4.6 \mathrm{~mm} \mathrm{Hg}$ (SBP), $4.6 \mathrm{~mm} \mathrm{Hg}$ (MAP), $4.3 \mathrm{~mm} \mathrm{Hg}$ (DBP) during $\mathrm{MA}_{\text {verbal }}$ and by $4.6 \mathrm{~mm} \mathrm{Hg}$ (SBP), $4.9 \mathrm{~mm} \mathrm{Hg}$ (MAP), $4.7 \mathrm{~mm} \mathrm{Hg}$ (DBP) during $\mathrm{MA}_{\text {nonverbal. }}$ The RT task did not affect the average blood pressure across the protocol.
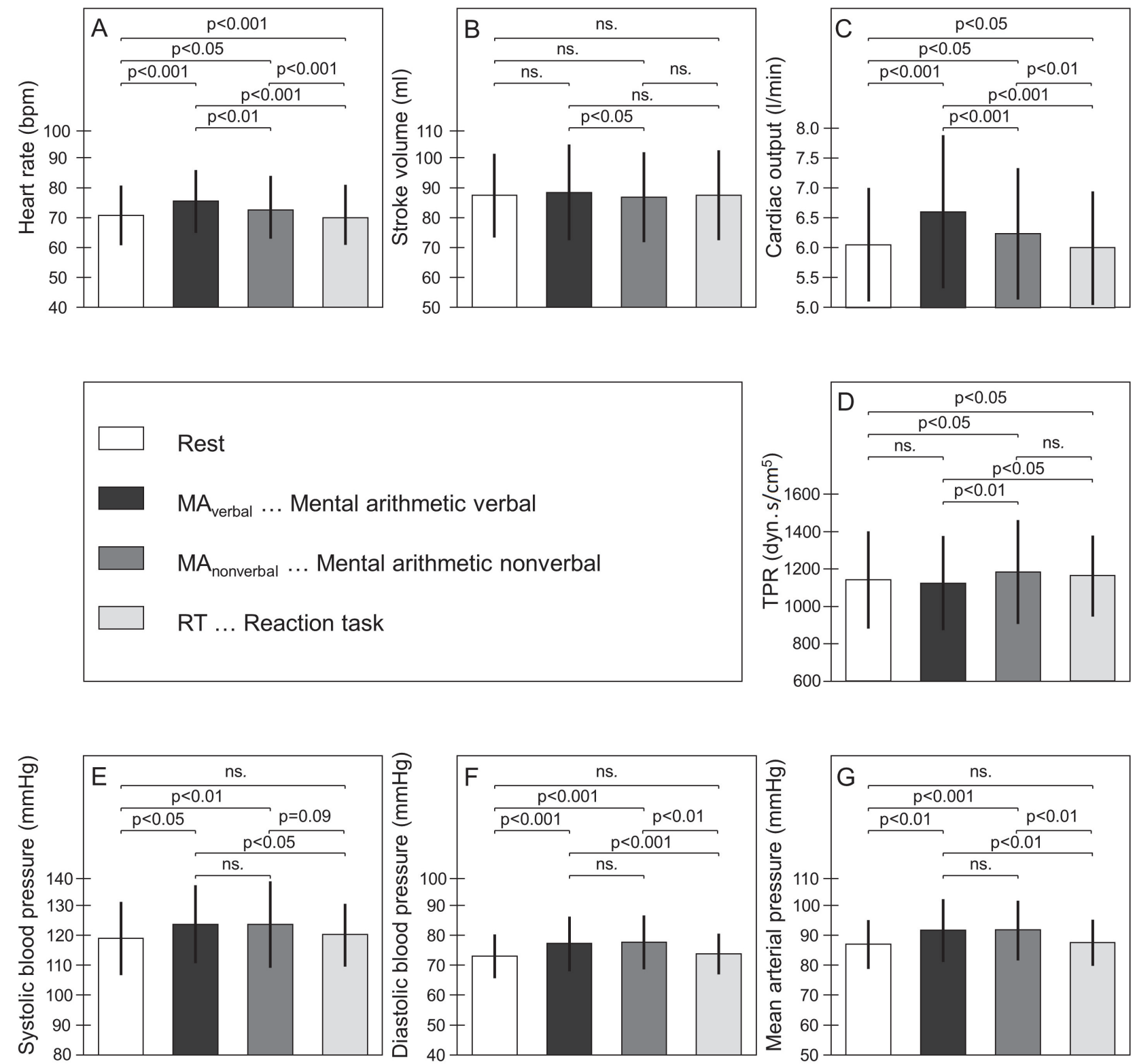

Fig. 3. Overall response of heart rate (HR; A), stroke volume (SV; B), cardiac output (CO; C), total peripheral resistance (TPR; D), systolic (E), diastolic (F) and mean arterial blood pressure (MAP; G; mean \pm SD) and Bonferroni corrected post-hoc tests. The order of the subplots refers to the relationship MAP HR $\times S V \times T P R$. 


\section{Respiration}

The ANOVAs indicated significant differences between the protocols for RF $(F(2.5,142.8)=12.7$, $\left.\mathrm{p}<0.001, \quad \eta_{p}{ }^{2}=0.18\right)$ and $\mathrm{SD}_{\mathrm{RF}} \quad(F(2.3,129.2)=62.5 .8$, $\left.\mathrm{p}<0.001, \eta_{p}{ }^{2}=0.52\right)$. On average, RF increased from $M=17.2 \mathrm{~min}^{-1}(S D=2.1)$ during the rest condition to $M=18.5 \mathrm{~min}^{-1}(S D=1.6)$ during $\mathrm{MA}_{\text {verbal }}$, and $M=18.5 \mathrm{~min}^{-1}$ $(S D=1.6)$ during $\mathrm{MA}_{\text {nonverbal; during } \mathrm{RT}} M=17.9 \mathrm{~min}^{-1}$ $(S D=2.4)$. $\mathrm{SD}_{\mathrm{RF}}$ was $M=2.9 \mathrm{~min}^{-1}(S D=1.0)$ at rest and increased during $\mathrm{MA}_{\text {verbal }} \quad(M=4.3, \quad S D=0.6)$ and $\mathrm{MA}_{\text {nonverbal }}(M=3.6, S D=1.0)$, whereas it decreased during RT $(M=2.7, S D=0.9)$. Bonferroni corrected post-hoc tests were significant for all differences.

\section{Heart rate variability}

SDNN, but none of the heart rate variability variables in the frequency domain $\left(\mathrm{LF}_{\mathrm{RRI}}, \mathrm{HF}_{\mathrm{RRI}}\right.$ and $\mathrm{LF} / \mathrm{HF}_{\mathrm{RRI}}$ ) differed between the protocols (SDNN, $F(2.5,143.3)=6.1, \quad \mathrm{p}<0.01, \quad \eta_{p}{ }^{2}=0.10 ; \quad \mathrm{LF}_{\mathrm{RRI}}$, $F(2.7,152.3)=1.2, \quad$ ns.; $\quad \mathrm{HF}_{\mathrm{RRI}}, \quad F(2.7,151.8)=0.4$, ns.; $\mathrm{LF} / \mathrm{HF}_{\mathrm{RRI}}(F(2.7,151.1)=1.2$, ns. $)$. SDNN did not differ between $\mathrm{MA}_{\text {verbal }}, \mathrm{MA}_{\text {nonverbal, }}$ and $\mathrm{RT}$. The normalized frequency components of the heart rate at $1 / 7.5 \mathrm{~Hz}$, which corresponds to the pace of stimulus presentation, were $M=0.6 \%, S D=0.6$ (rest), $M=3.7 \%, S D=3.7 \quad\left(\mathrm{MA}_{\text {verbal }}\right)$, $M=4.9 \%, S D=5.1\left(\mathrm{MA}_{\text {nonverbal }}\right), M=5.4 \%, S D=4.5(\mathrm{RT})$; $F(3,55)=42.7 ; \mathrm{p}<0.001, \eta_{p}{ }^{2}=0.70$. No differences were observed between the three task protocols.

\section{Transient Responses}

\section{Overall responses to the stressors}

In addition to the results of the overall reactivity (see Fig. 2), the analysis of the 1 min frames indicated differences in the time courses of the responses between the protocols during the stressor application for HR and $\mathrm{CO}$, as well as for the blood pressure variables. Means, standard deviations and statistics are given in Table 1. In addition, the time courses of the mentioned variables above differed between RT and $\mathrm{MA}_{\text {verbal }}$ and between RT and $\mathrm{MA}_{\text {nonverbal }}$ as can be seen in Table 1. However, the time courses did not differ between the two mental arithmetic protocols (Bonferroni corrected post-hoc tests).

\section{Initial responses to the stressors}

In the analysis of the $30 \mathrm{~s}$ frames from the task onset until $2.5 \mathrm{~min}$ after task start, the interaction Protocol $x$ Time was significant for $\mathrm{HR}, \mathrm{SV}, \mathrm{CO}$, and TPR, indicating differences in the time courses of the responses between the protocols during the initial time stressor application. Means, standard deviations and statistics are given in Table 2. Furthermore, the time courses of all mentioned variables above differed between RT and $\mathrm{MA}_{\text {verbal }}$ and between RT and $\mathrm{MA}_{\text {nonverbal }}$. The time courses did not differ between the two mental arithmetic protocols (Bonferroni corrected post-hoc tests).

The interaction Protocol $\mathrm{x}$ Time was significant in the analysis of the blood pressure variables. Please see Table 2 for means, standard deviations and statistics. During $\mathrm{MA}_{\text {verbal }}$ and $\mathrm{MA}_{\text {nonverbal, all blood pressure }}$ variables increased during the first minute of the task and remained on their elevated levels afterwards whereas during RT no significant changes of blood pressure were observed. As a result, the time courses of all mentioned variables above differed between $\mathrm{RT}$ and $\mathrm{MA}_{\mathrm{verbal}}$ and

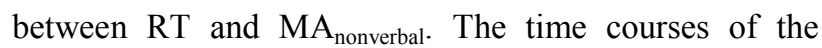
blood pressure variables did not differ between $\mathrm{MA}_{\text {verbal }}$ and $\mathrm{MA}_{\text {nonverbal }}$ (Bonferroni corrected post-hoc tests).

Means, standard deviations and statistics of the analysis of the $7.5 \mathrm{~s}$ frames for the first $30 \mathrm{~s}$ from task onset are given in Table 3. In contrast to the transient responses using time frames of $1 \mathrm{~min}$ and $30 \mathrm{~s}$ the time courses of the reported variables during the first $30 \mathrm{~s}-$ analyzed in steps of $7.5 \mathrm{~s}$ each - did not differ between the conditions. That is, the immediate initial transient response did not show different transient time course variations in $\mathrm{RT}, \mathrm{MA}_{\mathrm{verbal}}$, and $\mathrm{MA}_{\text {nonverbal }}$. Considering that transient time course variations reflect important features of the system response referring to dynamic control processes, it follows that the differences in the time courses between conditions observed in the $30 \mathrm{~s}$ frame were due to processes starting later.

The additional analysis of the $30 \mathrm{~s}$ frames from 2.5 min after task onset till the end of the task, that is, the prolonged effect of the stressor applied, showed compared to the analysis of the first $2.5 \mathrm{~min}$ after task onset (Table 2) a different result. While the main effect of Protocol was significant for $\operatorname{HR}(F(1.8,102.9)=23.1$, $\left.\mathrm{p}<0.001, \eta_{p}{ }^{2}=0.29\right)$ and $\mathrm{CO}(F(1.8,101.9)=13.0, \mathrm{p}<0.001$, $\left.\eta_{p}{ }^{2}=0.19\right)$ but not for $\mathrm{SV}(F(1.5,87.2)=0.3$, ns. $)$ and TPR $(F(1.7,98.1)=2.7$, ns. $)$, no significant interaction Protocol $\mathrm{x}$ Time were seen for the mentioned variables at all (HR, $F(6.7,382.1)=1.1$, ns.; SV, $F(5.3,304.0)=1.3$, ns.; CO, $F(3.5,197.1)=1.1$, ns.; TPR, $F(2.4,134.2)=0.5$, ns. $)$. That is, besides no difference in the time course between $\mathrm{MA}_{\text {verbal }}$ and $\mathrm{MA}_{\text {nonverbal}}$, the time courses of all mentioned variables above did not differ between RT and the mental arithmetic protocols. 


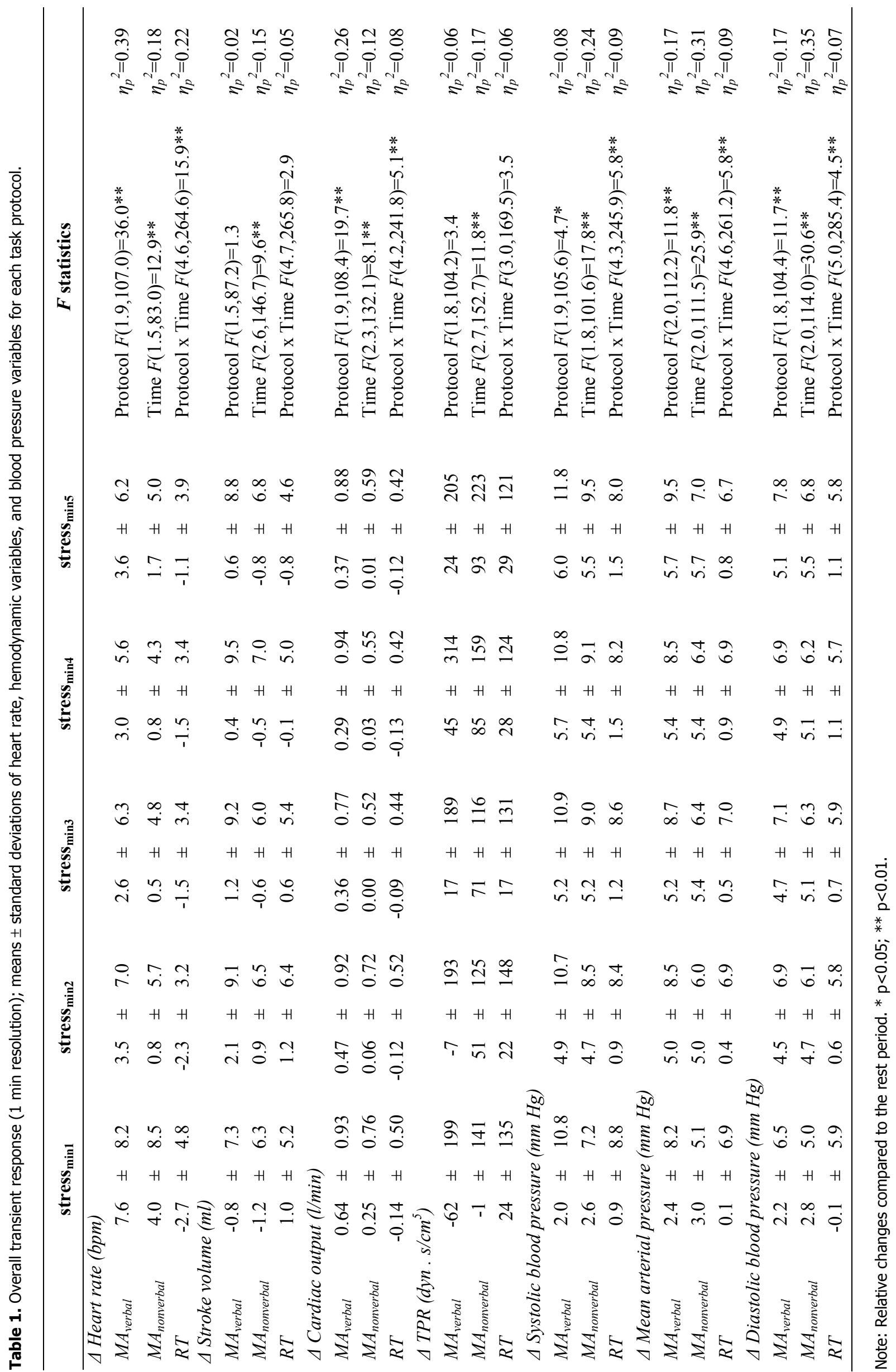




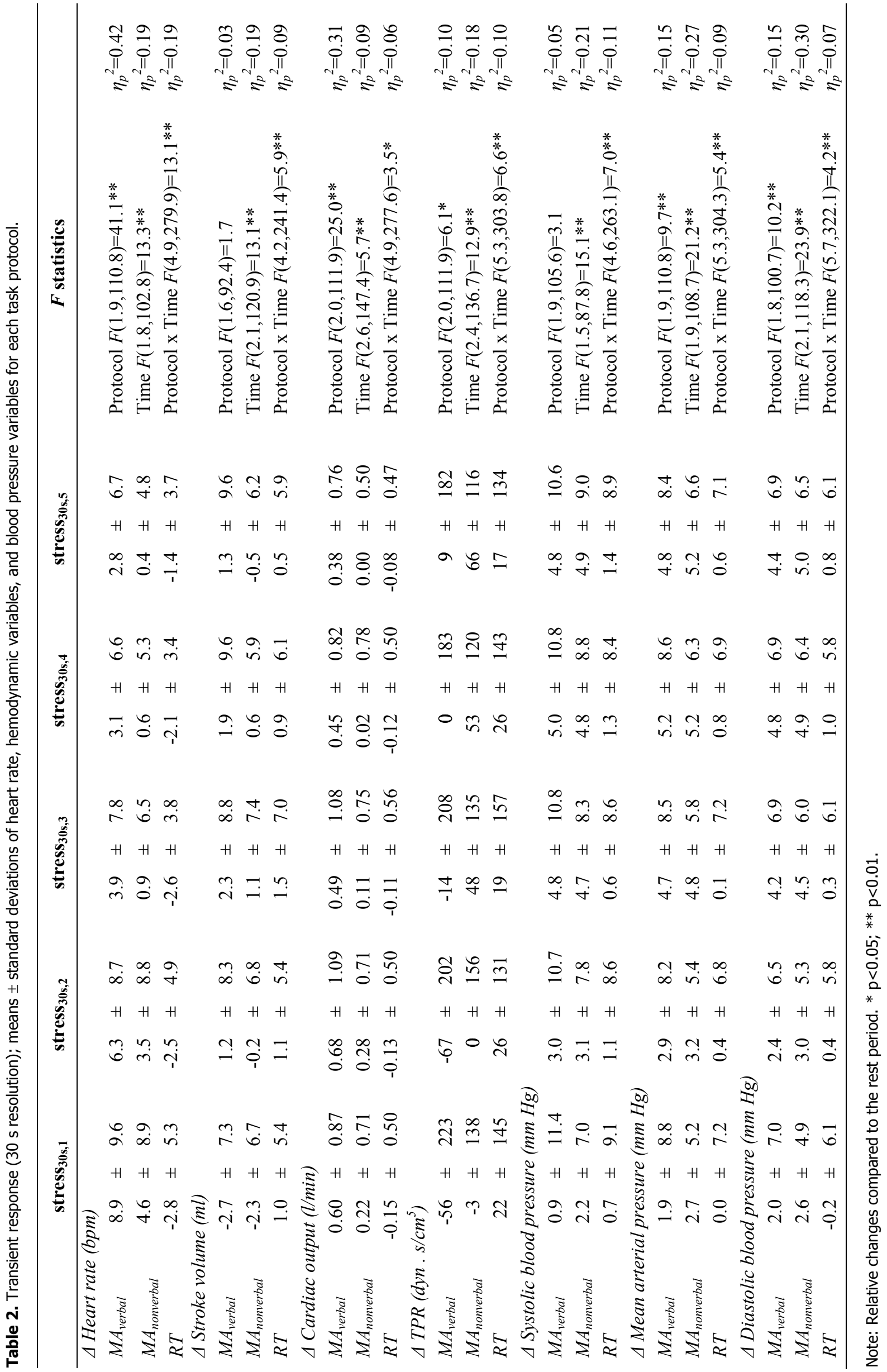




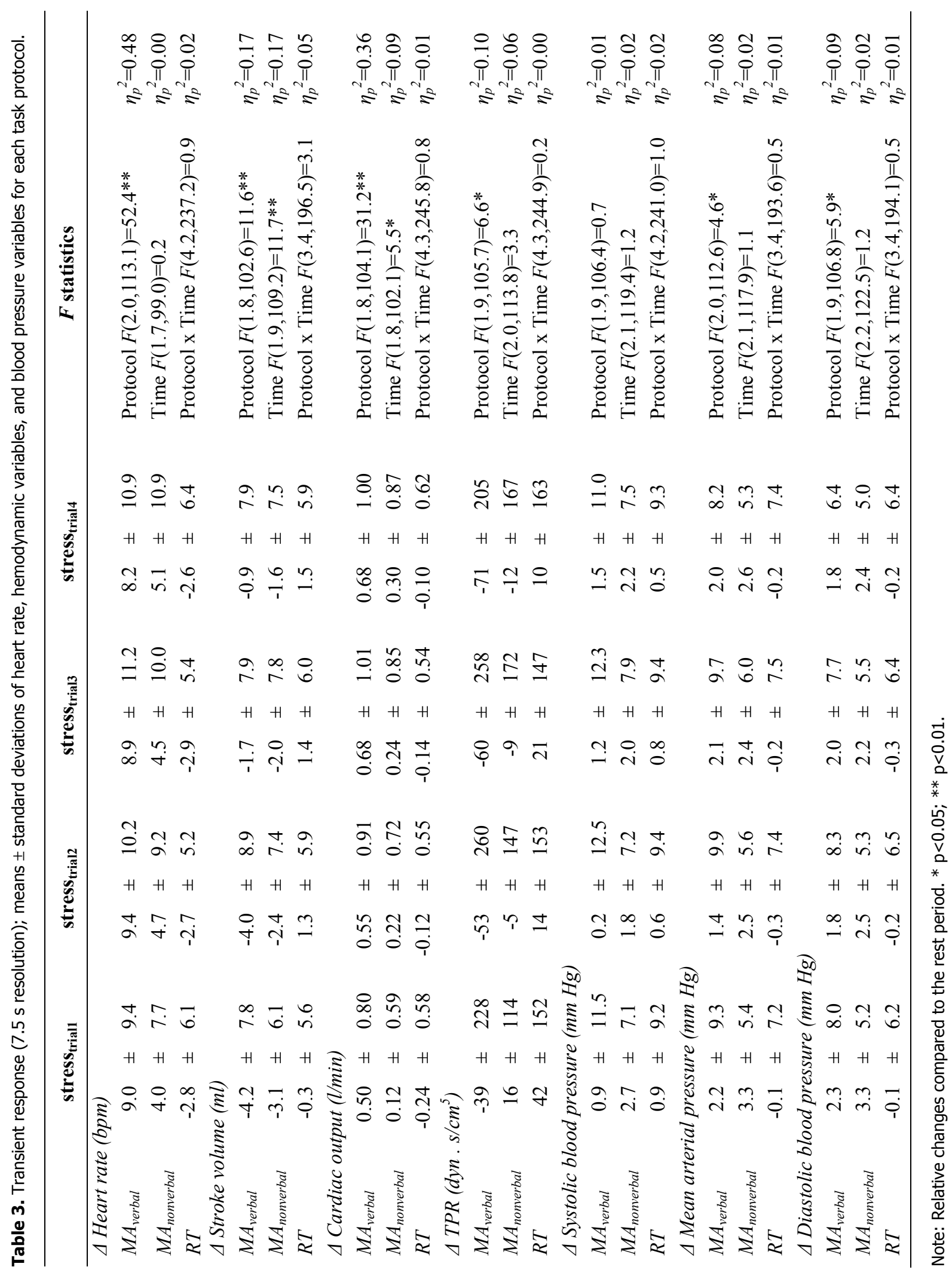




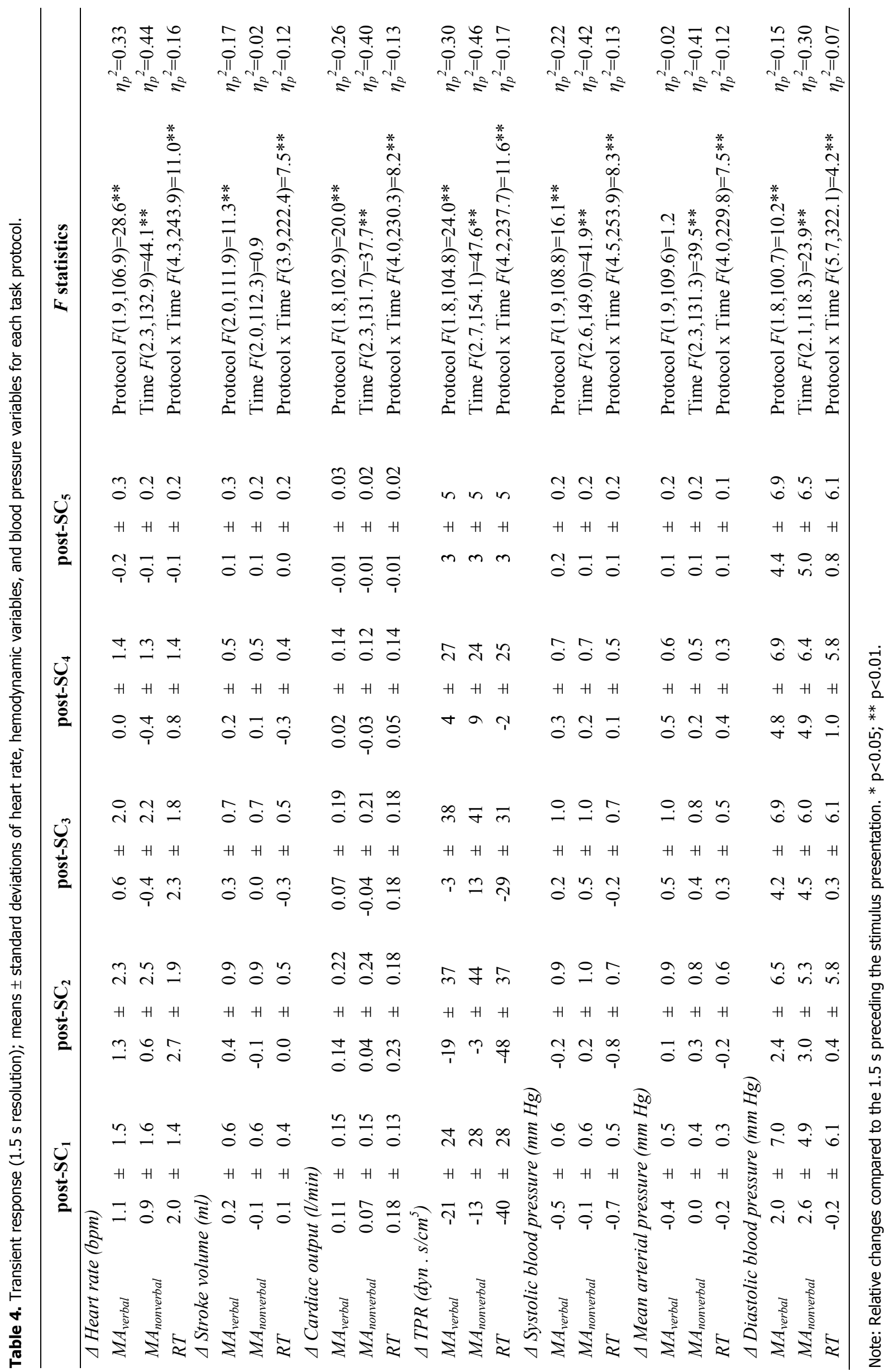


The analysis of the blood pressure variables showed a significant main effect of Protocol (SBP, $\quad F(1.9,106.9)=6.2, \quad \mathrm{p}<0.01, \quad \eta_{p}{ }^{2}=0.10 ; \quad$ MAP, $F(2.0,113.4)=13.1, \quad \mathrm{p}<0.001, \quad \eta_{p}{ }^{2}=0.19 ; \quad$ DBP, $\left.F(1.9,108.5)=12.5, \mathrm{p}<0.001, \eta_{p}{ }^{2}=0.18\right)$ but no significant interaction Protocol x Time (SBP, $F(5.9,338.1)=0.6$, ns.; MAP, $F(6.4,363.2)=1.0$, ns.; DBP, $F(6.5,373.1)=1.0$, ns. were observed as well.

\section{Immediate responses to stimulus presentation}

Means, standard deviations and statistics of HR, $\mathrm{SV}, \mathrm{CO}$, and TPR as well as the blood pressure variables are shown in Table 4. Bonferroni corrected post-hoc tests indicated different time courses of HR and SV during the two mental arithmetic tasks as compared to the RT task, but no differences between $\mathrm{MA}_{\text {verbal }}$ and $\mathrm{MA}_{\text {nonverbal }}$.

In the analysis of the fast transient responses of the blood pressure the interaction effects were significant for all variables.

\section{Supplemental analyses}

The number of correct answers was lower in the $\mathrm{MA}_{\text {nonverbal }}(M=32.3, S D=6.7)$ than in the $\mathrm{MA}_{\text {verbal }}$ task $\left(M=34.4, S D=5.6 ; F(1,56)=14.2, \mathrm{p}<0.001, \eta_{p}{ }^{2}=0.20\right)$. On average, the answer button was clicked after $M=5.0 \mathrm{~s}$ $(S D=0.8)$ in the $\mathrm{MA}_{\text {nonverbal }}$ task and $M=6.7 \mathrm{~s}(S D=0.1)$ after the beginning of a trial in the RT task (i.e. reaction time $M=0.7 \mathrm{~s}$ ). The motivation and difficulty ratings were lowest during the RT task (motivation $M=6.9, S D=5.5$, difficulty $M=2.5, S D=2.8$ ) compared to $\mathrm{MA}_{\text {nonverbal }}$ (motivation $M=11.1, S D=4.3$, difficulty $M=8.7, S D=4.1$ ) and $\mathrm{MA}_{\text {verbal }}$ (motivation $M=11.3, S D=4.3$, difficulty $M=9.0, \quad S D=4.2 ; \quad F(2,56)=29.2, \quad \mathrm{p}<0.001, \quad \eta_{p}{ }^{2}=0.51$; $\left.F(2,56)=53.6, \quad \mathrm{p}<0.001, \quad \eta_{p}{ }^{2}=0.66\right) . \quad$ Self-reported motivation and difficulty did not differ between the two mental arithmetic tasks. There was no decline over the course of the three task blocks of the experiment in how much effort the participants had made to accomplish the task $(F(2,56)=1.5$, ns. $)$ and how difficult they rated the task $(F(2,56)=0.4$, ns. $)$, indicating that variations of fatigue did not play an essential role in this experiment (e.g. Wright et al. 2011). The effort ratings $(M=10.2$, $S D=5.0 ; \quad M=10.1, \quad S D=5.2 ; \quad M=9.1, \quad S D=5.1)$ do also confirm that the participants were motivated throughout the experiment despite the absence of explicit incentives.

Although the order of tasks was counterbalanced, additional analyses for relative changes of HR, SBP, MAP, DBP, and RF for $\mathrm{MA}_{\text {verbal }}, \mathrm{MA}_{\text {nonverbal }}$ and RT compared to rest were performed. This was done to evaluate the potential influence of the order of occurrence, that is, if there was, for instance, a difference in the relative change of $\mathrm{HR}$ during $\mathrm{MA}_{\text {verbal }}$ depending on whether $\mathrm{MA}_{\text {verbal }}$ was the first, second or third task. No significant differences were observed.

\section{Discussion}

The distinctive feature of the present study using three tasks which involved varying degrees of task challenges is to demonstrate that the application of different levels of scales allows a more specific analysis of the factors and effects, that is, that different levels of scale reveal distinct effects.

\section{Overall reactivity}

In agreement with what has been reported (e.g. Callister et al. 1992, Gendolla and Richter 2006), the analysis of the overall activity during an entire protocol revealed that the mental arithmetic tasks increased HR, $\mathrm{CO}, \mathrm{BP}$, and $\mathrm{RF}$. $\mathrm{MA}_{\text {nonverbal }}$ represented a cognitive stressor, but had the same motor demands as the reaction task. Therefore, the differences in cardiovascular reactivity between the two tasks can be mainly attributed to the mental arithmetic aspects of the latter task, that is, the higher $\mathrm{HR}, \mathrm{CO}$ and $\mathrm{BP}$ in $\mathrm{MA}_{\text {nonverbal than in } \mathrm{RT}}$ reflected the mobilisation of mental effort.

As compared to $\mathrm{MA}_{\text {nonverbal, }}$ where the answers were given in private by selecting the correct answer on the computer monitor, $\mathrm{MA}_{\mathrm{verbal}}$ implied an additional social-evaluative element. It was clear to the participants that the verbal answers were monitored by the experimenter, placing social-evaluation threat on them. According to previous reports, social observation should lead to a general increase in autonomic nervous system activity (e.g. Al'Absi et al. 1997, Gendolla and Richter 2006). The different cardiovascular response to $\mathrm{MA}_{\mathrm{verbal}}$ as compared to $\mathrm{MA}_{\text {nonverbal, }}$ specifically the relative increase of $\mathrm{HR}$ and $\mathrm{CO}$, supports this finding. However, no differences between the two mental arithmetic protocols were observed in the blood pressure reactivity. This might be due to the modest difficulty of the applied tasks, reflected in the high rates of correct answers (Gendolla and Richter 2006). The on average better performance shown in $\mathrm{MA}_{\text {verbal }}$ than in $\mathrm{MA}_{\text {nonverbal }}$ supports the assumption that the introduction of the additional social stress component in $\mathrm{MA}_{\text {verbal }}$ had in fact made an impact. On the other hand, self-reported motivation failed to show a difference between the two 
mental arithmetic protocols. However, it has been pointed out that in the context of social stress tasks the differences between conditions may only be subtle and self-report measures not sensitive enough to produce reliable correlations (Schwerdtfeger 2004).

Thus, if one is specifically interested in the psychophysiological effects of certain cognitive demands, confounding effects of social-evaluative stress can be prevented by using responses that are given in private (e.g. motor rather than verbal responses, no presence of experimenters or surveillance cameras during performance of tasks). The effects of cognitive effort can be separated from the effects of giving motor responses by applying two protocols that involve exactly the same motor responses, but of which only one requires cognitive effort. However, researchers should be mindful of potential differences in motivation and difficulty between the tasks, which may also influence the responses to some extent.

In contrast to heart rate and hemodynamic variables, no differences in the frequency domain variables of HRV were found between the protocols. This is in contrast to the frequently reported increase of low frequency heart rate variability, a decrease of high frequency power, and/or an increase of the LF/HF ratio to stressors such as mental arithmetic and reaction tasks (e.g. Berntson and Cacioppo 2004), although other findings have also been reported (Taelman et al. 2011). The physiological basis for specific influences on HRV and heart rate may differ. HRV is mainly mediated by fluctuations of sympathetic and parasympathetic nerve traffic to the sinoatrial node. The HF component is generally associated with parasympathetic action whereas the LF component reflects to a degree the input of the sympathetic nervous system and also reveals information of vagal modulation (e.g. Task Force 1996, Taylor et al. 2001). By contrast, the heart rate may be a valid index of the net effects of sympathetic and parasympathetic inputs to the sinus node, which may have particular prognostic relevance (Goldberger 1999, Lahiri et al. 2008).

Equivocal findings for heart rate variability might suggest an additional argument for examining (also) transient psychophysiological processes. However, HRV variables are not suitable for transient analysis, because their computation requires longer time frames. Moreover, the common removal of the second order trend prior to the spectral analysis cancels out any transient processes in the data. But by using heart rate, continuous blood pressure, and hemodynamic variables, transient psychophysiological responses can be reliably observed and the system response can be illustrated. Nevertheless, the expansion by more sophisticated mathematical methods such as wavelet analysis may represent an important future development.

\section{Transient responses}

\section{Overall responses to the stressors}

In addition to the information provided by the averaged overall reactivity, the analysis of transient responses revealed the dynamics of the cardiovascular system. Additionally to the different levels of the cardiovascular values during $\mathrm{MA}_{\text {verbal }}$ and $\mathrm{MA}_{\text {nonverbal }}$ as compared to RT, the time courses indicating the dynamic control processes also differed between the mental challenge conditions and the reaction task. Furthermore, it should be noted that the blood pressure variables did not differ between the reaction task and the resting condition (Fig. 2) but showed different values during the mental challenge tasks, suggesting that they were driven by the steady increase of the blood pressure during the mental challenge tasks.

\section{Initial responses to the stressors}

The analysis of initial transient responses showed that all variables had different time courses during $\mathrm{MA}_{\text {nonverbal }}$ as compared to $\mathrm{RT}$, indicating that cognitive effort affects heart rate, stroke volume, cardiac output, blood pressure and total peripheral resistance. The analysis of the transient responses made clear that the observed differences in the overall reactivity between the protocols were for the most part due to the different initial responses to the stressor. This is in agreement with previous findings (Lackner et al. 2010b).

During $\mathrm{MA}_{\text {verbal }}$, the initial response was the highest among the protocols, which can be attributed to the social-evaluative component that may have diminished as the participants focused on the task at hand. If the response had mainly been due to verbalisation, the initial transient increase should have persisted. However, no differences between the tasks were observed in the response dynamic during the first $30 \mathrm{~s}$. That is, the levels the cardiovascular variables reached immediately after the task onset remained relatively constant throughout the first $30 \mathrm{~s}$.

The findings demonstrate that the analysis of the transient response provides relevant additional information which supplements the information provided by quantifying the average response across the entire task 
protocol. Overall reactivity measurements incorporate but obscure the transient variations due to the cardiovascular control processes. Transient time course measurements reflect these dynamic control processes and interactions among variables, providing important additional information of system response that is lost when only average values over the entire protocol are analyzed. Using step by step time resolution analysis as described above, cardiovascular dynamic system response can be captured including the complex interactions among variables that arise as the organism adapts to current system stress. This can be seen, e.g. in the different results using time frames of $30 \mathrm{~s}$ compared to $7.5 \mathrm{~s}$ (see Table 2 and Table 3). Such information can be useful even if the goal is to quantify reactivity over a total test period.

Further, it seems obvious (but is important to consider) that if stimuli only evoke mild activation, their effect will only be detected by analyzing the (relatively strong) transient initial responses, which will vanish in an average across a longer time frame. Similar applies to the use of standard approaches such as heart rate variability that implicate trend removal. Researchers may use the transient responses of certain cardiovascular variables to calculate difference scores (e.g. cognitive task minus simple reaction time task), in order to relate the specific effect of the cognitive challenge to individual difference variables or clinical diagnoses. This allows for a more clear interpretation of such correlations.

\section{Immediate responses to stimulus presentation}

Every measurement induces a back-action on the measured subject, that is, stimuli involved in carrying out an experiment have effects that may or may not be significant but need to be taken into account (Lackner et al. 2010a). In fact, the present data suggest that short term effects of stimulus presentation can determine longerterm averages. Consequently, the immediate responses to the presentation of stimuli may affect the interpretation of results, particularly if protocols differ in their stimulus configuration.

The analysis of the cardiovascular data revealed that the screen change indicating a new task item produced immediate responses of heart rate. These responses did not differ between $\mathrm{MA}_{\mathrm{verbal}}$ and $\mathrm{MA}_{\text {nonverbal }}$. They were higher in the simple reaction task than in the mental arithmetic tasks, but this may have been an aftereffect of pressing the button caused by the preparation of the motor response which was closer to the new stimulus in the reaction task than in the mental arithmetic tasks. As the values $1.5 \mathrm{~s}$ prior to the stimulus change were subtracted from those following the stimulus presentation to calculate the immediate response, the closer pressing the button may have shown up as a more pronounced response in the reaction task. Similarly, the differences in the immediate responses of the mean arterial blood pressure between the protocols can be explained by the lower average heart rate during RT. The mean arterial pressure can be estimated according to the relationship: $\mathrm{MAP} \sim \mathrm{TPR} \times \mathrm{SV} \times \mathrm{HR}$. Taking into account that human physiology reacts beat to beat, the fact that the response starts from a lower level (in terms of heart rate) in RT compared to the mental arithmetic tasks may explain the different time courses in the relative change of the systolic blood pressure. This may have caused different responses in the mean arterial pressure, which is the primary regulated variable. That is, the differences in the mean arterial pressure between the protocols may be attributed to the different temporal characteristics of the responses, caused by different initial values of the heart rate. Therefore, we suggest that additional psychological demands do not affect the immediate responses to stimulus presentation, in contrast to the overall responses across the entire task period and the initial transient responses to the task as were discussed above.

Nevertheless, the presentation of new stimuli does have pronounced immediate effects on heart rate and mean arterial pressure, as was also revealed by the analysis in the frequency domain (of the entire $300 \mathrm{~s}$ interval). The effects of the presentation of a new stimulus were observed in the frequency of $1 / 7.5 \mathrm{~Hz}$ representing the inter-stimulus interval of $7.5 \mathrm{~s}$ (the pace of stimulus presentation). No differences in these immediate effects were observed between the protocols, confirming the notion that additional psychological features of the protocol do not impact the immediate responses to the presentation of stimuli. But it also implies that even in the $\mathrm{MA}_{\text {verbal }}$ protocol, which had a marked effect, the hemodynamic measures were influenced by the effects of stimulus presentation. In a previous study, simple instructor commands during a mental load task had the same effect (Lackner et al. 2010a). As the immediate responses may impact measures in the frequency domain depending on the pace of stimulus presentation (in the present case measures of variability in the low-frequency range were affected), it seems advisable to consider the effects of paced stimuli when designing protocols or interpreting cardiovascular 
responses to conditions with constant stimulus intervals.

Taken together, the study showed how variations in the set-up of an experiment involving psychological tasks which could affect the cardiovascular system responses to the challenge can be identified by step by step analysis using different time resolutions. Both variations in the cognitive demands of a task and variations of the type of responses had clear effects, which can be disentangled in an experiment by using appropriate reference conditions. The present study therefore demonstrated the value of analyzing transient changes of hemodynamic variables that can reveal information that is important for interpreting effects but may be lost when only average values over the entire protocol are used as a representative of the system behavior and response. In addition, the high-resolution analysis of the hemodynamic variables showed the additional impact of stimuli directly involved in structuring the protocol. Thus, the study provides useful indications of how experiments involving cognitive demands may be set up and which time resolution of the analysis may be chosen in order to find clear answers to the specific research question and depending on the conclusions that are intended to be drawn.

\section{Conflict of Interest}

There is no conflict of interest.

\section{Acknowledgements}

The authors would like to thank Therese Wallner for her valuable help in data collection.

\section{References}

AL'ABSI M, BONGARD S, BUCHANAN T, PINCOMB GA, LICINO J, LOVALLO WR: Cardiovascular and neuroendocrine adjustment to public speaking and mental arithmetic stressors. Psychophysiology 34: 266-275, 1997.

BERNTSON GG, CACIOPPO JT: Heart rate variability: stress and psychiatric conditions. In: Dynamic Electrocardiography. M MALIK, AJ CAMM (eds), Futura, New York, 2004, pp 57-64.

BERNTSON GG, CACIOPPO JT, FIELDSTONE A: Illusions, arithmetic, and the bidirectional modulation of vagal control of the heart. Biol Psychol 44: 1-17, 1996.

CACIOPPO JT, ROURKE PA, MARSHALL-GOODELL BS, TASSINARY LG, BARON RS: Rudimentary physiological effects of mere observation. Psychophysiology 27: 177-186, 1990.

CALLISTER R, SUWARNO NO, SEALS DR: Sympathetic activity is influenced by task-difficulty and stress perception during mental challenge in humans. J Physiol 454: 373-387, 1992.

CODISPOTI M, BRADLEY MM, LANG PJ: Affective reactions to briefly presented pictures. Psychophysiology 38: 474-478, 2001.

ERNST JM, LITVACK DA, LOZANO DL, CACIOPPO JT, BERNTSON GG: Impedance pneumography: noise as signal in impedance cardiography. Psychophysiology 36: 333-338, 1999.

EUBANKS L, WRIGHT RA, WILLIAMS BJ: Reward influence on the heart: Cardiovascular response as a function of incentive value at five levels of task demand. Motiv Emot 26: 139-152, 2002.

FORTIN J, HABENBACHER W, HELLER A, HACKER A, GRÜLLENBERGER R, INNERHOFER J, PASSATH H, WAGNER CH, HAITCHI G, FLOTZINGER D, PACHER R, WACH P: Non-invasive beat-to-beat cardiac output monitoring by an improved method of transthoracic bioimpedance measurement. Comput Biol Med 36: 1185-1203, 2006a.

FORTIN J, MARTE W, GRUELLENBERGER R, HACKER A, HABENBACHER W, HELLER A, WAGNER CH, WACH P, SKRABAL F: Continuous non-invasive blood pressure monitoring using concentrically interlocking control loops. Comput Biol Med 36: 941-957, 2006 b.

GENDOLLA GH, RICHTER M: Cardiovascular reactivity during performance under social observation: the moderating role of task difficulty. Int J Psychophysiol 62: 185-192, 2006.

GOLDBERGER JJ: Sympathovagal balance: how should we measure it. Am J Physiol 276: H1273-H1280, 1999.

GRAHAM FK: Distinguishing among orienting, defense and startle reflexes. In: The Orienting Reflex in Humans. HD KIMMEL, EH VAN OLST, JF ORLEBEKE (eds), Erlbaum, Hillsdale, New York, 1979, pp 137-167. 
GRAHAM FK, CLIFTON RK: Heart-rate change as a component of the orienting response. Psychol Bull 65: 305-320, 1966.

GRAMER M, SARIA K: Effects of social anxiety and evaluative threat on cardiovascular responses to active performance situations. Biol Psychol 74: 67-74, 2007.

GRATZE G, FORTIN J, HOLLER A, GRASENICK K, PFURTSCHELLER G, WACH P, SCHÖNEGGER J, KOTANKO P, SKRABAL F: A software package for non-invasive, real-time beat-to-beat monitoring of stroke volume, blood pressure, total peripheral resistance and for assessment of autonomic function. Comput Biol Med 28: 121-142, 1998.

HOUTVEEN JH, GROOT PFC, DE GEUS EJC: Validation of the thoracic impedance derived respiratory signal using multilevel analysis. Int J Psychophysiol 59: 97-106, 2006.

IANI C, GOPHER D, LAVIE P: Effects of task difficulty and invested mental effort on peripheral vasoconstriction. Psychophysiology 41: 789-798, 2004.

KAMARCK TW, LOVALLO WR: Cardiovascular reactivity to psychological challenge: conceptual and measurement considerations. Psychosom Med 65: 9-21, 2003.

KELSEY RM, BLASCOVICH J, LEITTEN CL, SCHNEIDER TR, TOMAKA J, WIENS S: Cardiovascular reactivity and adaptation to recurrent psychological stress: The moderating effects of evaluative observation. Psychophysiology 37: 748-756, 2000.

LACKNER HK, GOSWAMI N, HINGHOFER-SZALKAY H, PAPOUSEK I, SCHARFETTER H, FURLAN R, SCHWABERGER G: Effects of stimuli occurring at regular intervals during mental stress on cardiovascular reactivity. J Psychophysiol 24: 48-60, 2010a.

LACKNER HK, GOSWAMI N, PAPOUSEK I, ROESSLER A, GRASSER EK, MONTANI JP, JEZOVA D, HINGHOFER-SZALKAY H: Time course of cardiovascular responses induced by mental and orthostatic challenges. Int J Psychophysiol 75: 48-53, 2010 b.

LACKNER HK, PAPOUSEK I, BATZEL JJ, ROESSLER A, SCHARFETTER H, HINGHOFER-SZALKAY H: Phase synchronization of hemodynamic variables and respiration during mental challenge. Int J Psychophysiol 79: 401-409, 2011.

LACKNER HK, WEISS EM, SCHULTER G, HINGHOFER-SZALKAY H, PAPOUSEK I: I got it! Transient cardiovascular response to the perception of humor. Biol Psychol 93: 33-40, 2013.

LAHIRI MK, KANNANKERIL PJ, GOLDBERGER JJ: Assessment of autonomic function in cardiovascular disease: physiological basis and prognostic implications. J Am Coll Cardiol 51: 1725-1733, 2008.

LANG PJ, GREENWALD MK, BRADLEY MM, HAMM AO: Looking at pictures: affective, facial, visceral, and behavioral reactions. Psychophysiology 30: 261-273, 1993.

LOVALLO WR: Stress and Health. Biological and Psychological Interactions. Sage, Thousand Oaks, 1997.

OBRIST PA: Cardiovascular Psychophysiology. Plenum, New York, 1981.

PAPOUSEK I, PAECHTER M, LACKNER HK: Delayed psychophysiological recovery after self-concept-inconsistent negative performance feedback. Int J Psychophysiol 82: 275-282, 2011.

PAPOUSEK I, SCHULTER G, WEISS EM, SAMSON AC, FREUDENTHALER HH, LACKNER HK: Frontal brain asymmetry and transient cardiovascular responses to the perception of humor. Biol Psychol 93: 114-121, 2013.

PARATI G, SAUL JP, DI RIENZO M, MANCIA G: Spectral analysis of blood pressure and heart rate variability in evaluating cardiovascular regulation: a critical appraisal. Hypertension 25: 1276-1286, 1995.

RAMÍREZ I, SÁNCHEZ MB, FERNÁNDEZ MC, LIPP OV, VILA J: Differentiation between protective reflexes: Cardiac defense and startle. Psychophysiology 42: 732-739, 2005.

RICHTER M, GENDOLLA GH: Incentive value, unclear task difficulty, and cardiovascular reactivity in active coping. Int J Psychophysiol 63: 294-301, 2007.

RICHTER M, FRIEDRICH A, GENDOLLA GH: Task difficulty effects on cardiac activity. Psychophysiology 45: 869-875, 2008.

SCHWERDTFEGER A: Predicting autonomic reactivity to public speaking: don't get fixed on self-report data! Int $J$ Psychophysiol 52: 217-224, 2004.

SEERY MD, WEISBUCH M, BLASCOVICH J: Something to gain, something to lose: the cardiovascular consequences of outcome framing. Int J Psychophysiol 73: 308-312, 2009. 
SMITH TW, NEALEY JB, KIRCHER JC, LIMON JP: Social determinants of cardiovascular reactivity: Effects of incentive to exert influence and evaluative threat. Psychophysiology 34: 65-73, 1997.

SILVESTRINI N, GENDOLLA GHE: Mood-regulative hedonic incentive interacts with mood and task difficulty to determine effort-related cardiovascular response and facial EMG. Biol Psychol 82: 54-63, 2009.

TAELMAN J, VANDEPUT S, VLEMINCX E, SPAEPEN A, VAN HUFFEL S: Instantaneous changes in heart rate regulation due to mental load in simulated office work. Eur J Appl Physiol 111: 1497-1505, 2011.

TASK FORCE of the European Society of Cardiology and the North American Society of Pacing and Electrophysiology: Heart rate variability: standards of measurement, physiological interpretation, and clinical use. Circulation 93: 1043-1065, 1996.

TAYLOR JA, MYERS CW, HALLIWILL JR, SEIDEL H, ECKBERG DL: Sympathetic restraint of respiratory sinus arrhythmia: implications for vagal-cardiac tone assessment in humans. Am J Physiol Heart Circ Physiol 280: H2808-H2814, 2001.

TURPIN G, SCHAEFER F, BOUCSEIN W: Effects of stimulus intensity, rise time, and duration on autonomic and behavioral responding: Implication for the differentiation of orienting, startle, and defense responses. Psychophysiology 36: 453-463, 1999.

WRIGHT RA, STEWART CC, BARNETT BR: Mental fatigue influence on effort-related cardiovascular response: Extension across the regulatory (inhibitory)/non-regulatory performance dimension. Int J Psychophysiol 69: 127-133, 2011.

WRIGHT RA, TUNSTALL AM, WILLIAMS BJ, GOODWIN JS, HARMON-JONES E: Social evaluation and cardiovascular response: an active coping approach. J Pers Soc Psychol 69: 530-543, 1995. 